\title{
Beyond immunomodulation: The regenerative role for regulatory $T$ cells in central nervous system remyelination
}

\author{
Veronique E. Miron ${ }^{1}$ (D) \\ Received: 25 April 2017 / Accepted: 27 April 2017 / Published online: 11 May 2017 \\ (C) The Author(s) 2017. This article is an open access publication
}

\begin{abstract}
Central nervous system regeneration after injury can occur in the form of remyelination, the reinstatement of myelin around axons which restores axon health and function. However, remyelination often fails in chronic neurological diseases, such as progressive multiple sclerosis. The lack of currently approved pro-remyelination therapies highlights the need to elucidate the cellular and molecular mechanisms underpinning this regenerative process. Whereas some $\mathrm{T}$ lymphocyte subsets such as Th1 and Th17 are implicated in inducing myelin injury, a recent study by Dombrowski et al. reveals a novel role for regulatory $\mathrm{T}$ cells $\left(\mathrm{T}_{\text {regs }}\right)$ in directly driving remyelination, independent of immunomodulation (Nat Neurosci doi:10.1038/nn.4528 2017)(Dombrowski et al., 2017). This study is summarized in this Bits and Bytes.
\end{abstract}

Keywords Remyelination $\cdot$ Oligodendrocyte $\cdot$ Multiple sclerosis $\cdot$ Regulatory T lymphocyte $\cdot \mathrm{CCN} 3 \cdot$ Regeneration . Myelin

Regeneration can occur efficiently in the central nervous system (CNS) in the form of remyelination, whereby new myelin is ensheathed around axons to reinstate trophic/ metabolic support and insulation for electrical impulse conduction. This process requires oligodendrocyte progenitor cells (OPCs) to migrate to areas of injury, proliferate, and differentiate into myelin-producing oligodendrocytes. Remyelination is limited or fails altogether in various neurological disorders,

Veronique E. Miron

vmiron@staffmail.ed.ac.uk

1 Medical Research Centre for Reproductive Health, The Queen's Medical Research Institute, The University of Edinburgh, 47 Little France Crescent, Edinburgh, Scotland EH16 4TJ, UK most prominently in progressive multiple sclerosis (MS), leading to axon dysfunction or loss. Although it is recognized that this failure largely reflects impaired oligodendrocyte differentiation (Kuhlmann et al., 2008), the mechanisms underpinning successful remyelination are still not fully understood. This highlights the importance of identifying the cells and molecules driving remyelination in order to develop effective regenerative therapies.

While it is now recognized that the innate immune system (i.e. macrophages) supports remyelination (Davies and Miron, 2016; Lloyd and Miron, 2016), the adaptive immune system (e.g. T lymphocytes) has historically been considered deleterious for oligodendrocytes/ remyelination. For instance, proinflammatory Th1 and Th17 T cells have direct cytotoxic effects on human OPCs in vitro (Moore et al., 2015) and reduce remyelination in vivo (Baxi et al., 2015). However, the impairment of remyelination following depletion of total CD4+ or CD8+ T cell populations (Bieber et al., 2003) points to the existence of a pro-regenerative $\mathrm{T}$ cell subset. Consistent with this postulate, a T cell presence in MS lesions is concurrent with ongoing remyelination. Indeed, a recent study by Dombrowski and Fitzgerald and colleagues (Dombrowski et al., 2017) revealed a novel pro-regenerative role for regulatory T cells $\left(\mathrm{T}_{\text {regs }}\right)$, demonstrating that these cells directly stimulate remyelination independent of immunomodulation.

Using a focal model of toxin-induced demyelination in the mouse spinal cord, where the temporal distinction between myelin damage and remyelination allows investigations of the regenerative process in isolation, $\mathrm{T}_{\text {regs }}(\mathrm{CD} 3+\mathrm{CD} 4+$ Foxp3+) were detected in lesions at the time of oligodendrocyte differentiation and remyelination initiation (Dombrowski et al., 2017). These cells were found to be required for remyelination, as their specific depletion in a Foxp3-driven diphtheria toxin receptor model (Foxp3-DTR) led to reduced numbers of oligodendrocytes and remyelinated axons 
(Dombrowski et al., 2017). This was rescued by supplementation with exogenous wildtype $T_{\text {regs }}$. The effects of $T_{\text {reg }}$ depletion were mirrored in a distinct demyelination model (Dombrowski et al., 2017), whereby select oligodendrocytes in the brain are killed via copper chelation by the cuprizone toxin, confirming that the role of $\mathrm{T}_{\text {regs }}$ in remyelination is not dependent on the mode of demyelination nor is it restricted to the spinal cord.

$\mathrm{T}_{\text {reg }}$ depletion in both models did not alter numbers of total oligodendrocyte lineage cells nor proliferating OPCs, suggesting effects of $\mathrm{T}_{\text {regs }}$ on the differentiation of OPCs into mature myelinating oligodendrocytes. Indeed, exposing brain explants to $\mathrm{T}_{\text {regs }}$ or their conditioned media enhanced oligodendrocyte differentiation, myelination, and remyelination, in comparison to non-polarized CD4+ T cells (Dombrowski et al., 2017). These effects were independent of immunomodulation of i) the peripheral immune system, as explants are devoid of a circulation, and ii) microglia and astrocytes, as effects were still observed when their inflammatory response to dissection had subsided (Dombrowski et al., 2017). A direct effect of $T_{\text {regs }}$ on OPCs was confirmed in vitro, where $T_{\text {reg }}$ conditioned media enhanced the differentiation of isolated OPCs and accelerated myelination in OPC-neuronal co-cultures (Dombrowski et al., 2017). Proteomic profiling of $\mathrm{T}_{\text {reg }}$ conditioned media was carried out to identify proremyelination factors, and identified high expression of the growth regulator CCN3 (Dombrowski et al., 2017). This factor has previously been shown to be involved in tooth regeneration (Wang et al., 2014), but never shown to be expressed either by $\mathrm{T}$ cells or during CNS regeneration. CCN3 was found to be a critical component of the beneficial effects of $\mathrm{T}_{\text {reg }}$ conditioned media, as use of a blocking antibody or specific depletion from the conditioned media abolished the prodifferentiation and pro-myelination effects (Dombrowski et al., 2017). Importantly, $\mathrm{T}_{\text {reg }}$-derived CCN3 was sufficient to support these responses (Dombrowski et al., 2017).

Altogether, these data demonstrate that $T_{\text {regs }}$ are direct drivers of oligodendrocyte differentiation and remyelination, thereby revealing a novel regenerative function for $T_{\text {regs }}$ beyond immunomodulation. Considering this study, one must now acknowledge that the adaptive immune system is not solely involved in damage induction and modulation of inflammation, but is also a critical component of the regenerative process that follows. A recent study demonstrated that MS patient-derived CD4+ T cells injected into demyelinated mouse CNS show high variability in their ability to support remyelination (El Behi et al., 2017), leading to the tantalizing possibility that the inter-patient variation in remyelination efficiency in MS might reflect diverging capacity of $\mathrm{T}_{\text {regs }}$ to stimulate remyelination. Overall, these studies support that further investigations into the pro-remyelination function of $\mathrm{T}_{\text {regs }}$ and CCN3 in MS are warranted for development of novel regenerative therapies.

Acknowledgements Veronique Miron is funded by a career development award from the Medical Research Council and United Kingdom Multiple Sclerosis Society, and research grants from Action Medical Research, United Kingdom Multiple Sclerosis Society, RS MacDonald Trust, GlaxoSmithKline and Biotechnology and Biological Sciences Research Council.

Open Access This article is distributed under the terms of the Creative Commons Attribution 4.0 International License (http:// creativecommons.org/licenses/by/4.0/), which permits unrestricted use, distribution, and reproduction in any medium, provided you give appropriate credit to the original author(s) and the source, provide a link to the Creative Commons license, and indicate if changes were made.

\section{References}

Bieber A, Kerr S, Rodriguez M (2003) Efficient central nervous system remyelination requires T cells. Ann Neurol 53:680-684

Baxi EG, DeBruin J, Tosi DM, Grishkan IV, Smith MD, Kirby LA, Strasburger HJ, Fairchild AN, Calabresi PA, Gocke AR (2015) Transfer of myelin-reactive th17 cells impairs endogenous remyelination in the central nervous system of cuprizone-fed mice. J Neurosci Off J Soc Neurosci 35:8626-8639

Davies CL, Miron VE (2016) Distinct origins, gene expression and function of microglia and monocyte-derived macrophages in CNS myelin injury and regeneration. Clin Immunol S1521-6616:3014230145

Dombrowski Y, O'Hagan T, Dittmer M, Penalva R, Mayoral SR, Bankhead P, Fleville S, Eleftheriadis G, Zhao C, Naughton M et al (2017) Regulatory T cells promote myelin regeneration in the central nervous system. Nat Neurosci 20:674-680

El Behi M, Sanson C, Bachelin C, Guillot-Noel L, Fransson J, Stankoff B, Maillart E, Sarrazin N, Guillemot V, Abdi H et al (2017) Adaptive human immunity drives remyelination in a mouse model of demyelination. Brain J Neurol 140:967-980

Kuhlmann T, Miron V, Cui Q, Wegner C, Antel J, Bruck W (2008) Differentiation block of oligodendroglial progenitor cells as a cause for remyelination failure in chronic multiple sclerosis. Brain $\mathrm{J}$ Neurol 131:1749-1758

Lloyd AF, Miron VE (2016) Cellular and molecular mechanisms underpinning macrophage activation during Remyelination. Front Cell Dev Biol 4:60

Moore CS, Cui QL, Warsi NM, Durafourt BA, Zorko N, Owen DR, Antel JP, Bar-Or A (2015) Direct and indirect effects of immune and central nervous system-resident cells on human oligodendrocyte progenitor cell differentiation. J Immunol 194:761-772

Wang X, He H, Wu X, Hu J, Tan Y (2014) Promotion of dentin regeneration via $\mathrm{CCN} 3$ modulation on notch and BMP signaling pathways. Biomaterials 35:2720-2729 